\title{
Pequeño Mal Impulsivo, Síndrome de Janz
}

\author{
Dr. Marcelo Devilat B. - Dra. Laura Germain P.
}

\author{
Impulsive Petit Mal.
}

\begin{abstract}
Four patients with Janz's "Impulsive Petit Mal" are described, with the characteristics of this disease, and its diferential diagnosis.

The etiology, prevalence, asociation with other types of epilepsy, were analized, making a paralel between reports in the literature and the data obtained from our patients.

Last of all, the critical and intercritical encefalografic characteristics, are described.
\end{abstract}

El Pequeño Mal Impulsivo (P.M.I.) fue descrito en el siglo pasado por varios autores (Heberden 1804, Herpin 1867, Rabot 1899$)^{1}$, con diversos nombres, hasta que Janz ${ }^{2-3}$ en 1955 to denomina Pequeño Mal Impulsivo. Por último Janz y Christian $^{4}$ delimitan la estructura clínica y electroencefalografía del cuadro.

Su prevalencia relativamente alta entre las Epilepsias en otros paises: $5 \%$ en adultos ${ }^{1-5}$ y $3 \%$ en niños ${ }^{6}$, justifica una llamada de atención sobre este cuadro, que es raramente diagnosticado en nuestro medio.

En esta presentación damos cuenta de 4 casos recopilados en menos de 6 meses en muestro Servicio. Durante los últimos meses, mientras redactábamos esta comunicación hemos identificado otros cuatro.

Consideramos de interés hacer una revisión del tema para motjvar un mejor conocimiento de la enfermedad.

\section{CASOS CLINICOS}

\section{CASO 1.-}

E.V.L. Niño de 9 años en el que se diagnostica Tumor de Tronco en 1979 por lo cual recibió por dos meses Cobaltoterapia y Oncovin ${ }^{\circledR}$ hasta Enero de 1981. Tiene antecedentes de asfixia al nacer y el parto con fórceps. El abuelo paterno y un hermano, sufren crisis de Gran Mal. Tres meses después de diagnosticado el tumor de tronco, el niffo presenta poco después de despertar por la mañana y después de dormir siesta, sacudidas y elevación

Servicio de Neurología y Psiquiatría. Hospital Luis Calvo Mackenna Departamento de Pediatría. Sede Oriente. Universidad de Chile. de los hombros, rápidas, violentas, como choque eléctrico, acompañadas de leve flexión de las extremidades inferiores sin compromiso de la cabeza. Estas sacudidas duran unos segundos, ocurren rítmicamente, aisladas o en salvas y el episodjo puede durar hasta 30 minutos. Durante él, el niño aduce los codos contra el cuerpo y coloca las manos en los bolsillos en un intento de controlar las descargas. Los episodios ocurren en forma diaria, a pesar de haber recibido varias combinaciones de antiepilépticos con el diagnóstico de convulsiones secundarias (Fenobarbital, Fenobarbital + Carbamazepina, Fenobarbital + Carbamazepina + Fenitoína).

De 4 EEG, uno es normal, 3 presentan desorganización lenta del ritmo basal en forma difusa.

Ante el cuadro clínico señalado, se plantea en Junio de 1981 Epilepsia Generalizada, Pequeño Mal Impuisivo probablemente secundario a Glioma de tronco. Se suspende la triple medicación que ingeria y se indica Valproinato de Magnesio con lo cual las sacudidas disminuyeron a los pocos días, de incontables en 30 minutos y todos los días, a solo 1 ó 2 por semana.

Tres meses después del diagnóstico de Pequeño Mal Impulsivo, el niño se hospitaliza para estudio de su cuadro de base y por razones que se ignoran, el Valproinato de Magnesio es suspendido con lo que reaparecen las crisis con una frecuencia de 30 por día. Dado de alta y controlado nuevamente, las crisis han reaparecido con igual frecuencia $e$ intensidad que antes del tratamiento. Reinstalado éste, las sacudidas desaparecen y está libre de ellas en los tres últimos meses.

\section{CASO 2.}

S.T.G. Consulta en 1979, a los 12 años de edad por haber presentado una crisis de Gran Mal. Entre 
sus antecedentes personales no aparecían anormalidades destacables. Un hermano suyo había tenido a los 2 años una convulsión febril. Se indica Fenobarbital y se solicita un EEG que revela desorganización lenta theta. Permanece sin crisis 2 affos, al cabo de los cuales repite crisis de Gran Mal sin relación horaria, por lo cual se agrega a la terapia, Fenitoina. Tres meses después ya no presenta crisis de Gran Mal, pero relata "leves estremecimientos matutinos con la mirada perdida, que duran segundos" y ocurren varias veces al día. En la prueba de hjperventilación habría presentado una ausencia compleja. Se diagnostica Petit Mal y se indica Fenobarbital, Ethosuxímida y Valproinato de $\mathrm{Mg}$. En un interrogatorio dirigido relata que, a los 12 años de edad comienza a presentar poco después de levantarse $y$ en la mafiana durante las clases, "estremecimientos", como sacudidas de los hombros, sin compromiso de conciencia, de 1 segundo de duración, hasta 6 al día, a veces en salva, sir fenómenos pre o postictales, pero acompañados de cierre de los párpados. Estos episodios ocurrian 2 a 3 veces por semana y desaparecieron completamente después de la introducción de Valproinato de Magnesio. Con el diagnóstico de Epilepsia Generalizada, crisis de Gran Mal, probables ausencias complejas y Pequeño Mal Impulsivo, se indica continuar con Fenobarbital y Valproinato de Magnesio.

Controlado en Octubre de 1981 , no ha vuelto a tener crisis de Gran Mal, ausencias ni Pequeño Mal Impulsivo, con lo cual completa 1 año libre de crisis.

\section{CASO 3.-}

M.T.A. Nifio de 13 años que consulta en Junio de 1978, por presentar en los últimos 15 dias, 2 crisis de Gran Mal. Entre sus antecedentes no figura patología pre, peri o postnatal. Su desarrollo sicomotor habia sido normal y no habia antecedentes de Epilepsia entre sus farniliares. Examen neurológico nomal. Con el diagnóstica de Gran Mal, Epilepsia Generalizada se indica Fenobarbital que recibe en forma irregular. Repite varias crisis de Gran Mal, por lo menos una ocurrió durante el sueño y 2 al despertar. Tiene tres EEG; en 2 de ellos ritmo de base normal, descargas de punta onda lenta 2.5 - 3.5 c.p.s. $\mathrm{Rx}$ cráneo y pruebas metabólicas normales. En mayo de 1981, interrogada la madre, relata que desde Diciembre de 1979 presenta, sin relación horaria, bruscas sacudidas de las 4 extremidades, consistentes en flexión de las inferiores y elevación de hombros, a veces con anteropulsión de la cabeza, violentas, como un rayo, golpe, o sobresalto, que compromete toda la extremidad. Ocurren todos los días, 1 a 2 veces al día, no hay compromiso de conciencia, sin pérdida del tono postural, sin aura ni fenómenos postictales. Cuando le aparecen estos episodios se le caen las cosas de las manos. En una oportunidad mientras trabajaba en una botillería al sobrevenirle la crisis dejó caer una caja de botellas por lo cual fue despedido. Se diagnostica Epilepsia de Gran Mal, Petit Mal Impulsivo agregándose al Fenobarbital, Valproato de Magnesio $600 \mathrm{mgrs}$. diarios.

Controlado 4 meses después, hab ra dejado de tener las sacudidas al poco tiempo de iniciar la ingesta de Valproato de Magnesio.

\section{CASO 4.-}

S.N.B. Niña de 8 afios de edad, que consulta en Abril 1981 por presentar en 3 oportunidades, poco después de despertar, crisis de Gran Mal.

Antecedentes personales y desarrollo psicomotor normales. Antecedentes familiares de una tía materna, 2 primos maternos y 1 primo materno portadores de Gran Mal. Una prima materna sufre ausencias del tipo Petit Mal. Examen neurológico normal.

Con el diagnóstico de Epilepsia Generalizada Gran Mal se inicia tratamiento con Fenitoína y se solicita nuevo EEG con deprivación de sueño que revela en el suer̂o, hiperventilación y fotoestimulación, descargas frecuentes de polipunta onda lenta de alto voltaje sincrónicas, simétricas y bilaterales con desorganización lenta theta, polimorfia difusa con predominio centrotemporal izquierdo.

Ante el trazado con polipunta-onda lenta, se interrogó dirigidamente a la madre y a la niña, quienes informan que desde Marzo 1981, presenta todos los días poco después de despertar en la mañana y al mediodía al regresar de clases, sacudidas bruscas, como "saltos" o "temblores" de los hom. bros y extremidades inferiores con pulsión de la cabeza hacia abajo. Ocurren en salvas 2 a 3 veces al dí, sin compromiso de conciencia, aura ni fenómenos postictales. Durante el episodio dejaba caer las cosas de las manos. Ocasionalmente ocurrian mientras escribia y una o dos rayas en la hoja eran la consecuencia del fenómeno ictal.

Se diagnostica Epilepsia del despertat, Gran MaI y Pequeño Mal Impulsivo. Se agrega a la Fenitoina, Valproinato de Magnesio con lo cual los episodies desaparecen a los pocos días.

Cuatro meses después de la introducción del Valproinato la niña no habia vuelto a tener ningún tipo de crisis.

\section{COMENTARIOS}

Los cuatro pacientes presentados, tienen en co- 
mún las siguientes caracter j́sticas:

- Sacudidas impulsivas, simétricas y sincrónicas de hombros y extremidades superiores e inferio. res, a veces también de la cabeza; bruscas, de cortísina duración (1 seg. - 2 seg.), descritas como sobresaltos, estremecimientos, golpe como choque eléctrico e involuntarias.

- Ocurren aisladas o en salva.

- Frecuencia diaria y núrnero variable de sacudidas, desde 1 o 2 al día hasta varias en 30 minutos.

- No se asocian a compromiso de conciencia, aura ni fenómenos postictales.

- Suceden especialmente después del despertar y en las mañanas.

- Se asocian con otras formas de epilepsia generalizada, gran mal del despertar y ausencias.

- Son frecuentes los antecedentes de epilepsia entre familiares de primer grado.

- Muestran excelente respuesta terapeútica a los derivados del ácido Valproico.

De acuerdo con sus caracter isticas clínicas estos pacientes son portadores del tipo de epilepsia denominado P.M.I.

EJ P.M.I., que se ubica entre las epilepsias ge. neralizadas, debe diferenciarse de algunas afecciones neurológicas que presentan descargas musculares similares:

La epilepsia mioclónica progresiva o enfermedad de Unverricht Lundborg, la disinergia cerebelar mioclónica o las mioclonías de cuadros degenerativos, la epilepsia parcial continua de Kojewnikow y la encefalopatía postanóxicca, ${ }^{6 \cdot 7 \cdot 8}$

En ellos el sintoma eje es la descarga mioclónica generalmente asimétrica y asincrónica, es decir, el compromiso de partes de fibras musculares o de un músculo o limitado grupo de ellos, situación que no se observa en el P.M.I. en que el fenómeno compromete simétrica y sincrónicamente grandes grupos musculares de las extremidades, produciendo desplazamiento de ellas. Por otra parte, en los cuadros antedichos, signos neurológicos progresivos como temblor, ataxia y alteración de la palabra acompañan siempre al síntoma mioclónico.

También debe considerarse el diagnóstico diferencial con las epilepsias focales motoras, pero en ellas el fenómeno muscular es generalmente lateralizado y con características tónicas, clónicas, o tónico-clónicas. Los espasmos masivos se pueden distinguir del P.M.I. por la edad de comienzo, siempre en lactantes. Por último, la forma ausencias complejas, con automatismos motores, mioclonías palpebrales, retropulsión de globos oculares y compromiso fugaz de conciencia deben ser considerados, sin embargo, en el P.M.I. el sintoma eje es la sacudida, corta, violenta y sin compromiso de conciencia.
Prevalencia (Tabla 1). E] 4.3\% (280/8.500) de los epilépticos adultos presentan P.M.I. ${ }^{1}$. Otros autores, en una muestra no seleccionada por edad señalan un $5.3 \%(399 / 7.400)^{5}$. En los niños, el cuadro sería menos frecuente, $3,1 \% 1$. En nuestra experiencia ${ }^{9}$, encontramos entre epilepticos adultos que el $4.5 \%(10 / 218)$ presentan el cuadro. Por último en una revisión que hicimos en 1978 no encontramos P.M.I. entre 100 nifos epilépticos esenciales que estudiamos ${ }^{10}$. Un mejor conocimiento del cuadro nos ha permitido diagnosticar 4 casos en 5 meses.

Tabla 1.

Prevalencia del pequeño mal impulsivo (PMD)

$\begin{array}{lcrl}\text { Autor } & \text { No Epilépticos } & \text { No PMI } & \% \\ \begin{array}{l}\text { Bamberger } \\ \text { Matthes 1959 }\end{array} & \text { Niños } & - & 3.1 \\ \begin{array}{l}\text { (Cit. Janz 69) } \\ \text { Janz 1969 }\end{array} & \text { 1169 Adultos y niños } & 280 & 4.3 \\ \text { Tsuboi 1976 } & \text { 7400 Adultos y niños } & 399 & 5.3 \\ \text { Devilat, Janz 1980 } & \text { 218 Adultos } & 10 & 4.5 \\ \text { Devilat, Taha 1978 } & \text { 100 Niños } & 0 & 0.0\end{array}$

Edad de comienzo (Tabla 2). La edad de comienzo del P.M.I. es, según la mayoría de los autores, alrededor de la pubertad $y$ adolescencia. Para Tsuboi $1977^{5}$ el $86 \%(343 / 349)$ de sus enfermos inician el cuadro antes de los 20 años y más del $2 / 3$ del total, al 70.2\% (280/399) lo hicieron entre los 12 y 19 años. Para Janz $(1969)^{1}$ el $85 \%$ de sus 139 pacientes (118) presentaron por primera vez el cuadro entre los 13 y 20 años.

E] $33,6 \%$ de los pacientes de una serie ${ }^{\theta}$ comen. zó sus sintomas antes de los 14 años. Por otra parte, otros autores ${ }^{11}$ encontraron que el $21.8 \%$ de sus 55 enfermos con P.M.l. inician la enfermedad entre los 9 y 14 años de edad.

Tabla 2.

Edad de comienzo pequeño mal impulsivo

$\begin{array}{cccl}\text { Años } & \text { No enfermos } & \% & \text { Autor } \\ 0-20 & 343 / 399 & 86.0 & \text { Tsuboi } 1977 \\ 0-14^{*} & 134 / 399 & 33.6 & \text { Tsuboi } 1977 \\ 0-14^{* *} & 12 / 55 & 21.8 & \text { Penin } 1979 \\ 12-19 & 280 / 399 & 70.2 & \text { Tsuboi } 1977 \\ 13-20 & 118 / 139 & 85.0 & \text { Janz 1969 } \\ 11-20 & 100 / 138 & 73.0 & \text { Lecasble*** } \\ 11-20 & 50 / 70 & 71.4 & \text { Mendilgharsu** }\end{array}$

*5 a 14, **9 a 14, ***Cit. Janz 69 
Asociación con otros tipos de convulsiones. De los 4 enfermos presentados, llama la atención que tres de ellos presentan además crisis de gran mal generalizado, (gran mal del despertar, gran mal difuso y gran mal y ausencias respectivamente). No es ésta una coincidencia ni una situación sorprendente, por cuanto la asociación con este tipo de crisis epilépticas es la regla: una casuística ${ }^{8}$, revela que sólo 10 de 399 (2.5\%) presentaron P.M.I. solo; el resto muy frecuentemente gran imal (381/399); $95 \%$. La mayoria de éstos, crisis del despertar $375 / 399(89.5 \%)$.

Pronóstico y desarrollo. En general el pronóstico es favorable ${ }^{11}$. En una investigación ${ }^{8}$, se observo que el 47.6\% (130/273) con P.M.I. estaban libres de crisis por un plazo de 2 a 5 años de observación.

Etiología. Tres de los 4 pacientes no tienen antecedentes personales que puedan explicar su cuadro. El cuarto niffo, portador de un glioma de tronco plantea la interrogante de si hay o no relación entre su P.M.I. y su patología básica tumoral. Sí llama la atención que sus crisis se iniciaron dos o tres meses después del diagnóstico del tumor y del inicio de la cobaltoterapia y del Oncovin, todos estos factores que podrían ser la causa de la Epilepsia, sin embargo la sorprendente mejoria con el tratamiento específico de ácido Valproico, pone un poco en duda que aquellos factores que, de estar actuando suponen una jut portante organicidad, ejerzan algún papel en la gênesis de su epilepsia.

En la literatura, por otra parte, hay consenso de que frecuentemente los enfermos de P.M.I. no tiene factores exógenos agregados ${ }^{1-5-6}$.

Sexo. Tres de nuestros pacientes corresponden al sexo masculino, uno al femenino. En la literatura no hay unanimidad, sin embargo, acerca de si hay alguna preponderancia de un sexo sobre otro en P.M.I. ${ }^{1-5}$.

Antecedentes de epilepsia en la familia (Tabla 3 y 4). Los enfermos portadores de P.M.I. tienen una relativa alta proporción de familiares cercanos y lejanos con epilepsia.

Algunos autores ${ }^{1}$ han comunicado que el $25 \%$ (70/280) de sus pacientes con P.M.I. tienen antecedentes de epilepsia entre los familiares. Otros 5 dan para esta eventualidad un $27.3 \%$ (87/319). Esta alta cifra de parientes epilépticos en P.M.I. es muy superior por ejemplo, a una muestra de epilépticos no seleccionada comunicada por el mismo autor ${ }^{5}$ que dio un $9.9 \%(46 / 466)$. La diferencia es significativa $(\mathrm{p}<0.001)$.
Este mismo investigador ${ }^{5}$, estudió 1618 parientes cercanos de 319 enfermos con P.M.I. y observó que el 33\% (21/638) de los padres de hijos con P.M.I. tenían epilepsia y que ésta era significativamente más frecuente $(p<0.05)$ entre las mađres $(15 / 319-4.7 \%)$ que entre los padres (6/319-1.9\%). Los hermanos presentaron epilepsia en un $4.4 \%$ (31/705) y no hubo diferencia entre hermanos y hermanas $(15 / 358-4.2 \%$ ws $16 / 347-4.6 \%)$. Sin embargo las hijas de padres con P.M.I. tuvieron significativamente más epilepsia $(\mathrm{p}<0.01)$ que los hijos (11/112-9.8\% vs 3/163-1.8\%). Si se observa el grupo total de pacientes, de nuevo se observa una significativa diferencia en favor del sexo femenino $[42 / 778-5.4 \%$ vs $24 / 840-2.9 \%(p<0.01)]$ y el total de parientes cercanos con epilepsia fue de $4.1 \%$ $(66 / 618)$. Esta preponderancia de parientes de se. xo femenino sobre el masculino, de epilépticos también ha sido observada por otros auto. res $5-12-13$.

Tabla 3.

Epilepsia entre parientes lejanos y cercanos de enfemos con pequeño mal impulsivo

$$
\text { Caso indice } \begin{aligned}
& \text { Enfermos con } \\
& \text { antecedentes }
\end{aligned}
$$

$\begin{array}{lrcr}\begin{array}{l}\text { Castells y } \\ \text { Mendilaharsu }\end{array} & 7058 . & 26 & 37.1 \% \\ \text { Janz } 1969 & 280 & 70 & 25.0 \% \\ \text { Tauboi } 1977 & 399 & 87 & 27.3 \%\end{array}$

*Contrales 46/466 - 9.9\%(P<0.001)

\section{Tabla 4.}

Epilepsia entre parientes de enfermos con pequeño mal ímpulsivo (Tsuboi 1977)

\begin{tabular}{|c|c|c|c|c|}
\hline \multirow[t]{2}{*}{ Parentesco } & \multirow[t]{2}{*}{ Masculino } & Fernenino & \multicolumn{2}{|c|}{ Significaciôn } \\
\hline & & $\%$ & $\%$ & \\
\hline Padres & $6 / 319-1.9$ & $15 / 319-4.7$ & $21 / 638-3.3$ & $p<0.05$ \\
\hline Herm & $15 / 358-4.2$ & $16 / 347-4.6$ & $31 / 705-4.4$ & $p>0.05$ \\
\hline Hijos & $3 / 163-1.8$ & $11 / 112-9.8$ & $14 / 275-5.1$ & $\mathrm{p}<0.01$ \\
\hline otal & $10-2.9$ & $42 / 778-5.4$ & $66 / 1618-4.1$ & $p<0.0$ \\
\hline
\end{tabular}

Algunos autores han observado cierta semejanza clínica entre los parientes de enfermos con P.M.J.: el $15 \%$ de 116 parientes epilépticos también hicieron P.M.I., como sus casos indices ${ }^{14}$. 
En nuestros enfermos, no encontramos semejanza clínica, pero si una fuerte tendencia a convulsionar entre los parientes. Sólo 1 no tenía antecedentes de epilepsia, pero el resto tenía parientes con gran mal, convulsiones febriles y ausencias. Destaca entre ellos, la paciente S.N.B. quiér tenía 5 parientes y todos del lado materno con epilepsia. Además 4 de esos cuatro eran de sexo femenino.

Electroencefalograma. Durante la crisis, el EEG es característico: un ritmo de base en general normal, interrumpido por descargas de complejos de poliespiga - onda lenta, de 3 a 5 ciclos por segundo, bilaterales, simétricas $y$ sincrónicas, a veces con acentuación en regiones centro-frontal. La duración del paroxismo eléctrico es siempre mayor en 4 a 6 segundos que la crisis clínica. Pueden también ocurrir sólo poliespigas.

El EEG tomado entre las crisis, revela en el 10\% de los casos alteraciones del ritmo de base ${ }^{l}$ y muy frecuentemente algún tipo de actividad irritativa, específica para epilepsia. Algunos ${ }^{3}$, la encontraron en el $92 \%$ de sus 47 casos, otros ${ }^{15}$, en el $83 \%$ de los 42 casos con P.M.J. que comunicó. Otros investigadores ${ }^{5}$ han dado cuenta que el $70.1 \%$ de sus 387 pacientes tuvieron un EEG específico irritativo, cifra significativamente mayor al $16.3 \%$ encontrada en un grupo de epilépticos no seleccionados. Por último vale la pena mencionar que caracteristicamente un alto número de enfermos, $30.1 \%(145 / 381)$ tienen el tipico EEG de poliespigas onda lenta, en tanto que los epilépticos no seleccionados lo presentan en solo $7.9 \%$ de los casos $(37 / 466)^{5}$.

En nuestros enfermos 2 de 4 tuvieron este último patrón y en 1 de ellos, sirvió cono clave del diagnóstico.

\section{RESUMEN}

Se presentan cuatro casos clínicos que corresponden a un tipo de epilepsia a la que Janz denomina "Pequeño Mal Impulsivo". Se describen las características de este cuadro planteándose el diagnóstico diferencial con otras causas de movimientos mioclónicos.

Se analiza etiologia, prevalencia, asociación con otras formas de Epilepsia, haciéndose un paralelo entre lo descrito en la literatura y los datos aporta. dos por nuestros pacientes.

Por último anotan las características encefalográficas críticas e intercríticas.

\section{REFERENCIAS}

1 Janz, D. Die Epilepsien, Spezielle Pathologie und Therapie. Georg Thierne Verlag. Stuttgart. 1969. Pág. 135.

2 Janz, D. Die Petit-Mal Epilepsien. Habilitationsschrift. Heidelberg. 1955.

3 Janz, 0 . Anfallsbild und Veriaufsforms epileptischer Erkrankungen. Nervenarzt 26: 20, 1955.

4 Janz, D., Christion, W. Impulsiv.Petit Mal. Dtsch. Z. Nerverheilk. 176: 346, 1957.

$\$$ Tsuboi, T. Primary Generalized Epilepsy with Sporadic Myoclonias of Myoclonic Petit Type. Georg Thieme Publishers. Stuttgart. 1977. Pág. 1, 69, 97-99, 18-20, 51-96, 19-51.

6 Motrhes, A. Epilepsie. Diagnostik und Therapie für Klinik un Praxis. Georg Thieme Verlag. Stuttgart. 1977. Pág. 56.

${ }^{7}$ Hirt, H.R. MylokJonien. Klinick und Systematik der Myoklonien. Aktuelle Neuropädiatrie. 2. Jahrestagung der Gesellschaft für Neuropädiatrie. Kiel. Hermann Doose. Georg Thieme Verlag. Stuttgart. 1977. Pág. 1.

${ }^{a}$ Degert, $R$. Die Kindlichen Anfallsleiden. Epileptische und nichtepileptische Anfäle. Hippokrates Verlag.
Stuttgart. 1976. Pág. 123.

${ }^{9}$ Devilht, $M$. . Janz, D. Semejanzas y diferencias electroencéfalográficas entre padres epilépticas y sus hijos. Berlin Occidental 1980. En preparación.

${ }^{10}$ Devilat, M., Toha, L. Epilepsia infantil criptogenética I, II. Pediatría 21: 204, 1978.

11 Penin, H. Massiver bilateraler Myoklonus. Beiträge zur Klassifikation und medikamentösen Therapie epileptischer Anfälle. Hermann Doose u.a. Desitin-Work Carl Klinke Gmbh. Hamburg. 1979. Pág. 105.

12 Ounsted, $C$. The factor of inheritance in convulsive disorders in chidhood. Proc. Roy. Soc. Med. 45: 865, 1952.

13 Harvald, B. On the genetic prognosis of epilepsy. Acta psychiat. Scand 26:339, 1951.

14 Doose, H. Gerken, H., Hien-Völpel, KF. and Vökke, $E$. Genetics of photosensitfve epilepsy. Newropadiatrie 1: $56,1969$.

Is Aigter, B.R. and Mulder, D. W. Myoclonus. Arch. Neutol. 2: $600,1960$. 